\title{
Abemaciclib/Tamoxifen Regimen
}

National Cancer Institute

\section{Source}

National Cancer Institute. Abemaciclib/Tamoxifen Regimen. NCI Thesaurus. Code C160523.

A reg imen consisting of abemaciclib and tamoxifen that can be used for the treatment of prostate cancer. 